\title{
DNA barcode reference libraries: Achievements of the DNAqua-Net Working Group 1
}

\author{
Torbjørn Ekrem, Fedor Čiampor Jr§ \\ ‡ NTNU University Museum, Trondheim, Norway \\ $\S$ Plant Science \& Biodiversity Centre, Slovak Academy of Sciences, Bratislava, Slovakia
}

Corresponding author: Torbjørn Ekrem (torbjorn.ekrem@ntnu.no)

Received: 22 Feb 2021 | Published: 04 Mar 2021

Citation: Ekrem T, Čiampor Jr F (2021) DNA barcode reference libraries: Achievements of the DNAqua-Net Working Group 1. ARPHA Conference Abstracts 4: e64811. https://doi.org/10.3897/aca.4.e64811

\begin{abstract}
The three main aims for the DNAqua-Net working group 1 (WG1) have been i) to identify gaps in the barcode reference libraries of European aquatic species, ii) to fill these gaps in collaboration with ongoing initiatives, and iii) to facilitate knowledge transfer and initiate work on barcode reference libraries in regions where such activity was low (Leese et al. 2016). WG1 currently has 129 members from 39 countries spanning four continents, with a majority of members from Europe.

In our presentation we show the achievements of WG1 during the lifetime of the DNAquaNet COST action (CA15219; 2016-2021), and discuss some of the highlights obtained through collaborative work and joint dissemination. Specifically, we emphasize the outcomes presented in two peer-reviewed scientific publications that focus on standards for building DNA barcode reference libraries, and gaps in reference databases for European aquatic organisms currently used in national biomonitoring programs (Rimet et al. 2021, Weigand et al. 2019). However, major achievements were also obtained in four workshops (Braga, Pécs, Limassol \& Bratislava) and through the activities of 16 short-term scientific missions (STSMs).
\end{abstract}

\section{Keywords}

DNAqua-Net, WG1, DNA barcode, reference libraries 


\section{Presenting author}

Torbjørn Ekrem, Fedor Čiampor Jr

\section{Presented at}

1st DNAQUA International Conference (March 9-11, 2021)

\section{References}

- $\quad$ Leese F, Altermatt F, Bouchez A, Ekrem T, Hering D, Meissner K, Mergen P, Pawlowski J, Piggott JJ, Rimet F, Steinke D, Taberlet P, Weigand A, Abarenkov K, Beja P, Bervoets L, Björnsdóttir S, Boets P, Boggero A, Bones AM, Borja Á, Bruce K, Bursić V, Carlsson J, Čiampor F, Čiamporová-Zatovičová Z, Coissac E, Costa F, Costache M, Creer S, Csabai Z, Deiner K, DelValls Á, Drakare S, Duarte S, Eleršek T, Fazi S, Fišer C, Flot J, Fonseca V, Fontaneto D, Grabowski M, Graf W, Guðbrandsson J, Hellström M, Hershkovitz Y, Hollingsworth P, Japoshvili B, Jones J, Kahlert M, Kalamujic Stroil B, Kasapidis P, Kelly M, Kelly-Quinn M, Keskin E, Kõljalg U, Ljubešić Z, Maček I, Mächler E, Mahon A, Marečková M, Mejdandzic M, Mircheva G, Montagna M, Moritz C, Mulk V, Naumoski A, Navodaru I, Padisák J, Pálsson S, Panksep K, Penev L, Petrusek A, Pfannkuchen MA, Primmer C, Rinkevich B, Rotter A, Schmidt-Kloiber A, Segurado P, Speksnijder A, Stoev P, Strand M, Šulčius S, Sundberg P, Traugott M, Tsigenopoulos C, Turon X, Valentini A, van der Hoorn B, Várbíró G, Vasquez Hadjilyra MI, Viguri J, Vitonytè I, Vogler A, Vrålstad T, Wägele W, Wenne R, Winding A, Woodward G, Zegura B, Zimmermann J (2016) DNAqua-Net: Developing new genetic tools for bioassessment and monitoring of aquatic ecosystems in Europe. Research Ideas and Outcomes 2 https://doi.org/10.3897/rio.2.e11321

- $\quad$ Rimet F, Aylagas E, Borja A, Bouchez A, Canino A, Chauvin C, Chonova T, Jr FČ, Costa F, Ferrari BD, Gastineau R, Goulon C, Gugger M, Holzman M, Jahn R, Kahlert M, Kusber W, Leese F, Leliaert F, Mann D, Marchand F, Méléder V, Pawlowski J, Rasconi S, Rougerie R, Schweizer M, Trobajo R, Vivien R, Weigand A, Witkowski A, Zimmermann J, Ekrem T (2021) Metadata standards and practical guidelines for specimen and DNA curation when building barcode reference libraries for aquatic life. Metabarcoding and Metagenomics In press.

- Weigand H, Beermann A, Čiampor F, Costa F, Csabai Z, Duarte S, Geiger M, Grabowski M, Rimet F, Rulik B, Strand M, Szucsich N, Weigand A, Willassen E, Wyler S, Bouchez A, Borja A, Čiamporová-Zat'ovičová Z, Ferreira S, Dijkstra K, Eisendle U, Freyhof J, Gadawski P, Graf W, Haegerbaeumer A, van der Hoorn B, Japoshvili B, Keresztes L, Keskin E, Leese F, Macher J, Mamos T, Paz G, Pešić V, Pfannkuchen DM, Pfannkuchen MA, Price B, Rinkevich B, Teixeira ML, Várbíró G, Ekrem T (2019) DNA barcode reference libraries for the monitoring of aquatic biota in Europe: Gap-analysis and recommendations for future work. Science of the Total Environment 678: 499-524. 